\title{
Applying Sociocultural Models of Aging to Promote Optimal Health and Wellbeing Amongst Aging Populations: A Literature Review on Interdisciplinary Approaches to Mental Health Care
}

\author{
Dor David Abelman \\ School of Health Studies, Faculty of Health Sciences, Western University, Canada
}

*Correspondence to: Dor David Abelman, School of Health Studies, Faculty of Health Sciences, Western University, Arthur and Sonia Labatt Health Sciences Building, Room 222, London, N6A 5B9, Ontario, Canada; Email: dabelman@uwo.ca

Received: October 25, 2017; Accepted: November 15, 2017; Published: December 12, 2017;

\begin{abstract}
With a significant increase in aging populations in the coming years affecting many factors from increased health spending to restructuring of public spaces, challenges of how society can adapt to this major population change persist [1-4]. Sociocultural models of aging have been shown widely effective in a variety of contexts to describe processes of aging, theories to guide future experiments, and applications of theories into practice [5-7]. A review describing Bronfenbrenner's ecological perspective, the PPCT model, and the life course perspective is presented. Applications to research in the field of mental health gerontology, future aging research, and criticisms of theories are described. There is a focus on depression, dementia, treating the whole person, and cultural competency. Considering the variety of factors that influence an individual's risk and experience in living with a mental health issue, including their lived experience and personal preferences, holds important considerations for promoting optimal health and well-being in aging successfully.
\end{abstract}

Key Words: Gerontology, theory integration, social determinants of health, life course model, PPCT model, ecological perspective, dementia, depression, resiliency, positive psychology

\section{Introduction}

With a significant change in population demographics supporting a shift in aging populations already underway and only projected to increase in the coming years, it is important to consider how society can prepare for the coming change [1-4]. Significant modifications to healthcare spending and structuring are projected, in addition to modification of public spaces, among other factors [4-8]. Mental health remains an important factor affecting health, wellbeing, and aging, that effects populations around the world [8]. It is possible that one in five adults around the world may experience a mental health issue at least one time each year, and approximately one in three adults experience a mental health issue at least once in their adult life [9].

The World Health Organization asserts that approximately 15\% of older adults (aged 60 or older) around the world experience a mental health disorder [8]. Out of this population, approximately one in two older adults who experience a mental health issue experience a form of depression [8]. The symptoms of depression, along with other mental health issues in older adults such as substance use disorder, are often overlooked and untreated as they coincide with other health issues or are assumed to be just a part of the aging process [8]. A significant proportion of older adults experience dementia [8]. In 2016, it was estimated that approximately 47.5 million people worldwide live with dementia, and that this number is expected to triple by 2050 [8].

There are a variety of factors affecting the risk of the common mental health disorders of depression and dementia widely reported in different fields [10-21]. For instance, one can attribute risk to genetics, environmental interactions, social support, and lifestyle [10, $13,15,18-21]$. Although there is a significant representation of ways to address these diseases in different areas of literature, there is continued disagreement between fields on best practices, the actual weight and effects of different risks, and implementation of strategies to address them [13, 20-22]. There is a need for application of fundamental theory and models to help connect varying perspectives of factors influencing risks of experiencing mental health issues throughout life that can be readily used and applied by healthcare practitioners and Gerontological researchers. Models like these can help consolidate various findings from different fields to assist healthcare workers consider the multiple factors affecting one's experience with a mental health issue. When applied appropriately, a healthcare worker may be encouraged to think with a mindset that promotes acknowledging a person who is affected with a mental health issue's individual differences to put them on the path of best recovery options. Two models are presented below that discuss these issues. 


\section{Explanation and Application of Models}

\section{Bronfenbrenner's ecological perspective}

A major theory that can be applied to the topic of factors affecting health as they ages is Bronfenbrenner's ecological perspective [5, 2326]. The theory describes that an individuals development is strongly related to the environments they experience over time on a variety of levels, including micro-, meso-, exo-, macro-, and chrono-systems $[5,25,26]$. These systems include anything from political systems to the community, mass-media, family, health agencies and nationality, which all work together over time to influence life decisions and affect health $[5,25,26]$. Another component of the theory is the factors of process, person, context, and time, that form the acronym for the modern version of this theory, referred to as the PPCT model $[5,26]$. Process refers to the proximal interactions an individual has with their environment, such as participating in a hobby they enjoy or attending a social event $[5,26]$. Person refers to the biological and personality characteristics of an individual which affects the way they perceive the world $[5,26]$. This relates to their genetics and lived experience $[5,26]$. Context refers to the events occurring around an individual, such as their environment, culture, place of origin, employment, and interactions $[5,26]$. Time refers to when the interactions an individual has with their environment is occurring $[5,26]$. Furthermore, the theory promotes that events early in life can have important effects on health later in life [23-25].

Bronfenbrenner's ecological perspective is especially relevant when applying theories of social determinants of health to aging populations. The findings that ecological factors continue to be essential for health and wellbeing are promoted through a variety of studies such as the Whitehall II study and other reviews [27, 28]. In the Whitehall II study, social determinants of health such as income, socioeconomic status, and grade of employment were significantly correlated with health behaviours such as smoking and nutrition, the risk of health issues such as obesity and cardiovascular disease, and overall mortality in a longitudinal cohort study of over 10,000 individuals [27]. Data from this study continue to make new research findings affecting gerontology, such as the association between lower social class, increased body mass index (BMI), and increased cases of dementia amongst older adults [11]. The effect of having a high BMI even at age 50, but not 60 or 70, increased risk of having dementia in one study based on the findings from the Whitehall II cohort [11]. Other studies based on the findings of the Whitehall II study on social determinants of health continue to show varying effects of environmental factors on wellbeing later in life such as through predicting the risk of experiencing disability [29].

General criticisms of this theory are that it is not used appropriately when applied to research methodology [5]. Many studies who say they include the theory in their theoretical framework simply take from the theory that an individual's environment affects their health and do not include the other factors, such as timing and personality characteristics, in their discussion [5]. These components are an essential part of the theories' new development to the PPCT model [5]. By ignoring these aspects and referring to the theory's new form, authors commonly misrepresent in their work [5]. Another criticism may be the overemphasis of environmental factors influencing an individual's mental health, such as in the case of developing dementia, which can be largely attributed to other factors such as genetic vulnerability $[12,13,22]$. As Bronfenbrenner's theory includes preexisting personality and genetic traits in assessing determinants of health, (the 'person' part of the PPCT model), this criticism is invalid $[5,26]$. The theory, in its newer PPCT model form, is still relevant to diseases that have large genetic or pre-existing risk factors $[5,26]$. Whether the diseases have significant or minor preexisting risk factors does not disregard the continued need to discuss other factors such as lived experience $[5,26]$.

\section{The life course perspective}

The life course perspective promotes that norms, roles, and attitudes at any age all contribute to shaping a person's health and life choices $[6,7,30]$. It supports the ecological perspective in the support that events early in life can influence events later in life $[6,7,30]$. Some important parts of the model include pathways or trajectories of health behaviors, critical or sensitive periods of development such as at an important psychological phase of development or experience of trauma, and the cumulative impact of all these experiences [6]. Social, historical, and biological factors affecting age are all considered important in shaping the course of a person's health [30]. A healthcare practitioner following this model can look into these various factors over time to 'treat the whole person' instead of just the biological symptoms which may present, and builds upon a holistic model of Geriatric mental health care $[6,7,30,31]$.

The life course model has important implications when applied to the mental health of older adults. A review applying the theory to principles of adequate nutrition finds that consistently good nutrition across different stages of childhood development is important to promote optimal growth, health, and development throughout the lifespan [7]. Another review finds that cumulative effects of stressors relating to caregiving can lead to significantly negative psychological, social, and physiological effects for caregiver after a prolonged time [32]. Other studies suggest the loss or separation of a child from a parent can have psychological effects across the lifespan, including increased risk of experiencing depression and substance use issues which may continue through older adulthood [33-35]. This way of thinking has also been newly applied in other contexts affecting other age groups, such as how a variety of experiences over the course of life can influence the risk of young adults using drugs [36]. These different studies from various fields reflect on the importance of considering lived experiences and individual differences to help a healthcare worker determine the best course of treatment.

A criticism of the life course perspective is that it places too much emphasis on how experiences in early life can affect health later in life in a way that undermines resiliency [6]. Resilience is defined by the American Psychological Association as the ability to adapt to adversity, trauma, and tragedy and "bounce back" to normal [37]. Other authors describe it as the ability to have better outcomes than could be expected after a change in or negative circumstance [38]. A review of literature shows that older adults can be very resilient, even more than young adults, and that this has positive health implications [39]. 
Other studies suggest that high resiliency may be rare among older adults, but that having a strong social support network significantly increases the likelihood of experiencing it, and that this ultimately improves health outcomes [40]. A systematic review on quality of life of caregivers with dementia suggests resiliency can be associated with an increase in self-efficacy, which can has positive health effects on caregivers [41]. Another study suggests resiliency has important health effects for informal caregivers of people living with dementia, and that learning about resilience and how to recognize caregivers with low levels of it is important to improve their health and wellbeing [38]. Applying the life course perspective in a way that acknowledges an individual's lived experience without making them feel like they are unable to change can help address this potential issue.

\section{Discussion}

As a variety of fields continue to show different main risk factors or best practices for treatment of common mental health issues in aging populations, the amount of older adults in society is increasing significantly, and the proportion of older adults who experience some mental health issues such as dementia is increasing significantly, it is important to evaluate best practices for care. The ecological perspective (also referred to as the PPCT model) and life course perspective emphasize the importance of a variety of factors across the lifespan that influence risks of experiencing a mental health issue in later adulthood, and multidimensional strategies to best address them. When applied in practice, they can be used as a framework which consolidates findings from different fields relating to the causes and course of disease and acknowledging their varying factors of genetics and environment. Furthermore, these perspectives support the growing trend of 'treating the whole person' and individual treatment plans when discussing the causes and best methods of recovery relating to mental health care.

Some examples of ways that these models can be applied are in encouraging a practitioner to acknowledge an individual's culture when assessing what would be the best way to help their client. They may find that a variety of cultures do not support talking openly about their mental health issues, or would prefer to speak about them in a specific context which acknowledges their lived experience [42]. Thinking in this way may encourage a large proportion of individuals who may need help most, such as those from ethnic minorities who do not feel comfortable reaching out for help, seek treatment [42]. For those who do seek treatment, considering personalized lived experience in conjunction with traditional pharmaceutical practices relating to mental health care may lead to considerably improved treatment outcomes $[31,42,43]$.

Consideration of how various experiences throughout one's life contributes to their sense of meaning, happiness, or suffering is also described in the field of positive psychology [44]. The general shift in psychology away from solely treating an individual based on their symptoms and towards a more-encompassing interdisciplinary approach is encompassed by this field [44]. This general shift takes the focus away from individual experiences and looks towards a life course perspective of how a variety of factors all come together to influence mental health and wellbeing [44]. The authors of a review chapter on positive psychology, Seligman and Csikszentmihaly, cite a number of studies that suggest aspects of the life course and ecological perspectives are important for helping communities flourish [44]. Experiences of happiness, self-determination, subjective wellbeing, and optimism may all be related to and promoted through an interdisciplinary life course approach similar to the theories described above [44].

\section{Conclusion}

As the burden of mental health issues continues to increase amongst aging populations worldwide, methods of treating them become more sophisticated and comprehensive through increased research on best practices. There is a growing support to acknowledge a variety of factors affecting health and wellbeing throughout the life course as described in the life course and ecological perspectives, as well as the PPCT model, research on cultural competency, and the field of positive psychology. The trend of considering interdisciplinary events throughout life that influence health and wellbeing represents a general shift in psychology towards treating the whole person through considering the many factors that lead them to where they are today. When these interdisciplinary factors are considered, especially among aging populations, one can work towards promoting optimal mental health and wellbeing in a more effective way.

\section{References}

1. Dall TM, Gallo PD, Chakrabarti R, West T, Semilla AP, et al. [2013] An aging population and growing disease burden will require a large and specialized health care workforce by 2025. Health Aff (Millwood) 32: 2013-2020. [Crossref]

2. US Census Bureau (2017) An Aging Nation. Census Infographics \& Visualizations.

3. Rowe JW, Fulmer T, Fried L (2016) Preparing for Better Health and Health Care for an Aging Population. JAMA 316: 1643-1644. [Crossref]

4. Loukaitou-Sideris A, Levy-Storms L, Chen L, Brozen M. (2016) Parks for an Aging Population: Needs and Preferences of Low-Income Seniors in Los Angeles. J Am Plan Assoc 82: 236-251.

5. Tudge JRH, Payir A, Merçon-Vargas E, Cao H, Liang Y, et al. (2016) Still Misused After All These Years? A Reevaluation of the Uses of Bronfenbrenner's Bioecological Theory of Human Development. J Fam Theory Rev 8: 427-445.

6. U.S. Department of Health and Human Services (2010) Health Resources and Services Administration, Maternal and Child Health Bureau. Rethinking MCH: The Life Course Model as an Organizing Framework.

7. Herman DR, Taylor Baer M, Adams E, Cunningham-Sabo L, Duran N, et al. (2014) Life Course Perspective: Evidence for the Role of Nutrition. Matern Child Health J 18: 450-461. [Crossref]

8. World Health Organization (2016) Mental health and older adults. Fact Sheets.

9. Steel Z, Marnane C, Iranpour C, Chey T, Jackson JW, et al. (2014) The global prevalence of common mental disorders: a systematic review and meta-analysis 1980-2013. Int J Epidemiol 43: 476-493. [Crossref]

10. Belvederi Murri M, Amore M, Menchetti M, Toni G, Neviani F, et al. (2015) Physical exercise for late-life major depression. Br J Psychiatry 207: 235-242. [Crossref]

11. Singh-Manoux A, Dugravot A, Shipley M, Brunner EJ, Elbaz A, Sabia S, et al. (2017) Obesity trajectories and risk of dementia: 28 years of follow-up in the Whitehall II Study. Alzheimer's Dement. 2017. [Crossref]

12. Adams HHH, de Bruijn RFAG, Hofman A, Uitterlinden AG, van Duijn CM, et al. (2015) Genetic risk of neurodegenerative diseases is associated with mild cognitive impairment and conversion to dementia. Alzheimer's Dement 11: 1277-1285. [Crossref]

13. Bellou V, Belbasis L, Tzoulaki I, Middleton LT, Ioannidis JPA, et al. (2017) Systematic evaluation of the associations between environmental risk factors and dementia: An umbrella review of systematic reviews and meta-analyses. Alzheimer's Dement 13: 406-418. [Crossref]

14. Jacka FN, Pasco JA, Mykletun A, Williams LJ, Hodge AM, et al. (2010) Association of Western and traditional diets with depression and anxiety in women. $\mathrm{Am} \mathrm{J}$ Psychiatry 167: 305-311. [Crossref] 
Dor David Abelman (2017) Applying Sociocultural Models of Aging to Promote Optimal Health and Wellbeing Amongst Aging Populations: A Literature Review on Interdisciplinary Approaches to Mental Health Care

15. Jacka FN, Cherbuin N, Anstey KJ, Butterworth P (2014) Dietary patterns and depressive symptoms over time: Examining the relationships with socioeconomic position, health behaviours and cardiovascular risk. PLoS One 9. [Crossref]

16. Blumenthal JA, Babyak MA, Doraiswamy PM, Watkins L, Hoffman BM, et al. (2007) Exercise and pharmacotherapy in the treatment of major depressive disorder. Psychosom Med 69:587-596. [Crossref]

17. Bellamy S, Hardy C (2015) Understanding Depression in Aboriginal Communities and Families. Prince George, BC, Canada.

18. Erickson KI, Voss MW, Prakash RS, Basak C, Szabo A, et al. (2011) Exercise training increases size of hippocampus and improves memory. Proc Natl Acad Sci US A 108: 3017-3022. [Crossref]

19. Josefsson T, Lindwall M, Archer T (2014) Physical exercise intervention in depressive disorders: Meta-analysis and systematic review. Scand J Med Sci Sports 24: 259-272. [Crossref]

20. Pedersen BK, Saltin B (2015) Exercise as medicine - evidence for prescribing exercise as therapy in 26 different chronic diseases. Scand J Med Sci Sports 25: 1-72. [Crossref]

21. Flint J, Kendler KS (2014) The genetics of major depression. Neuron 81: 484-503. [Crossref]

22. Rodrigue KM, Rieck JR, Kennedy KM, Devous MD Sr, Diaz-Arrastia R, et al. (2013) Risk factors for $\hat{\mathrm{I}}^{2}$-amyloid deposition in healthy aging: vascular and genetic effects. JAMA Neurol 70: 600-606. [Crossref]

23. Bronfenbrenner U (1986) Ecology of the family as a context for human development: Research perspectives. Dev Psychol 22: 723-742.

24. Bronfenbrenner U (1977) Toward an experimental ecology of human development. Am Psychol 32: 513-531.

25. Bronfenbrenner U (1994) Ecological models of human development. Int Encycl Educ 3: 37-43.

26. Rosa EM, Tudge J (2013) Urie Bronfenbrenner's Theory of Human Development: Its Evolution From Ecology to Bioecology. J Fam Theory Rev 5: 243-258.

27. Marmot MG, Smith GD, Stansfeld S, Patel C, North F, et al. (1991) Health inequalities among British civil servants: the Whitehall II study. Lancet (London, England) 337: 1387-1393. [Crossref]

28. Allen J, Balfour R, Bell R, Marmot M (2014) Social determinants of mental health. Int Rev Psychiatry 26: 392-407. [Crossref]

29. Artaud F, Sabia S, Dugravot A, Kivimaki M, Singh-Manoux A, et al. (2016) Trajectories of Unhealthy Behaviors in Midlife and Risk of Disability at Older Ages in the Whitehall II Cohort Study. Journals Gerontol Ser A Biol Sci Med Sci 71: 1500-1506. [Crossref]

30. Elder GH, Rockwell RC (1979) The Life-Course and Human Development: An Ecological Perspective. Int J Behav Dev 2: 1-21.

31. Branch WT (2014) Treating the whole patient: passing time-honoured skills for building doctor-patient relationships on to generations of doctors. Med Educ 48: 67-74. [Crossref]

32. Bevans M, Sternberg EM (2012) Caregiving burden, stress, and health effects among family caregivers of adult cancer patients. JAMA 307: 398-403. [Crossref]

33. Nickerson A, Bryant RA, Aderka IM, Hinton DE, Hofmann SG (2013) The impacts of parental loss and adverse parenting on mental health: Findings from the National Comorbidity Survey-Replication. Psychol Trauma Theory, Res Pract Policy 5: 119-127. [Crossref]

34. Otowa T, York TP, Gardner CO, Kendler KS, Hettema JM (2014) The impact of childhood parental loss on risk for mood, anxiety and substance use disorders in a population-based sample of male twins. Psychiatry Res 220: 404-409. [Crossref]

35. Kuerbis A, Sacco P, Blazer DG, Moore AA (2014) Substance abuse among older adults. Clin Geriatr Med 30: 629-654. [Crossref]

36. Abelman DD (2017) Mitigating risks of students use of study drugs through understanding motivations for use and applying harm reduction theory: a literature review. Harm Reduct J 14: 68. [Crossref]

37. American Psychological Association (2017) The road to resilience.

38. Joling KJ, Windle G, Dröes RM, Huisman M, Hertogh CMPM, et al. (2017) What are the essential features of resilience for informal caregivers of people living with dementia? A Delphi consensus examination. Aging Ment Health 21: 509-517. [Crossref]

39. MacLeod S, Musich S, Hawkins K, Alsgaard K, Wicker ER (2016) The impact of resilience among older adults. Geriatr Nurs 37: 266-272. [Crossref]

40. Netuveli G, Wiggins RD, Montgomery SM, Hildon Z, Blane D (2008) Mental health and resilience at older ages: bouncing back after adversity in the British Household Panel Survey. J Epidemiol Community Heal 62: 987-991. [Crossref]

41. Crellin NE1, Orrell M, McDermott O, Charlesworth G (2014) Self-efficacy and health-related quality of life in family carers of people with dementia: a systematic review. Aging Ment Health 18: 954-969. [Crossref]

42. Betancourt JR, Green AR, Carrillo JE, Owusu Ananeh-Firempong I (2003) Defining Cultural Competence: A Practical Framework for Addressing Racial/Ethnic Disparities in Health and Health Care. Public Health Rep 118: 293-302. [Crossref]
43. Kidd S, Kenny A, McKinstry C (2015) The meaning of recovery in a regional mental health service: an action research study. $J A d v$ Nurs 71: 181-192. [Crossref]

44. Seligman MEP, Csikszentmihalyi M (2014) Positive Psychology: An Introduction. In: Flow and the Foundations of Positive Psychology. Dordrecht: Springer Netherlands. pp: 279-298.

\section{Citation:}

Dor David Abelman (2017) Applying Sociocultural Models of Aging to Promote Optimal Health and Wellbeing Amongst Aging Populations: A Literature Review on Interdisciplinary Approaches to Mental Health Care. Ageing Sci Ment Health Stud Volume 1(2): 1-4 